\title{
Foot Digit 2
}

National Cancer Institute

\section{Source}

National Cancer Institute. Foot Digit 2. NCI Thesaurus. Code C52840.

The second toe from the medial side of the foot. 